\title{
Determination of energetics and kinetics from single-particle intermittency and ensemble-averaged fluorescence intensity decay of quantum dots
}

\author{
Jau Tang ${ }^{\mathrm{a}, \mathrm{b})}$ and R. A. Marcus ${ }^{\mathrm{a}), \mathrm{c})}$ \\ Noyes Laboratory of Chemical Physics, California Institute of Technology, Pasadena, California 91125
}

(Received 13 December 2005; accepted 21 June 2006; published online 25 July 2006)

\begin{abstract}
Quantification of energetics and kinetics for the band-edge exciton states of quantum dots and the long-lived dark state is important for better understanding of the underlying mechanism for single-particle intermittency and ensemble fluorescence intensity decay. Based on a multistate diffusion-reaction model by extending our previous studies, we analyze experimental data from ensemble measurements and fluorescence intermittency of single quantum dots and determine important molecular-based quantities such as Stokes shift, free energy gap, activation energy, reorganization energy, and other kinetic parameters. (C) 2006 American Institute of Physics.
\end{abstract}

[DOI: $10.1063 / 1.2227394]$

\section{INTRODUCTION}

Recent development of nanofabrication techniques ${ }^{1-3}$ and single-molecule spectroscopy ${ }^{4,5}$ has opened a new realm of research and has offered many potential applications of these low-dimensional materials. Taking semiconductor nanocrystals, or quantum dots (QDs), as an example, properties such as tunable band-edge emission, very narrow linewidth, and large quantum yield have presented potential applications in optoelectronic devices ${ }^{1-3}$ and biological labeling. ${ }^{6}$

There are intensive studies of photophysical properties of QDs such as band-edge exciton states, ${ }^{7-11}$ intermittency, ${ }^{12-23}$ and theoretical modeling. ${ }^{24-32}$ Other than semiconductor nanoparticles, intermittency has also been observed in single-enzyme activity of biomolecules ${ }^{33,34}$ and in single organic chromophores embedded in a matrix. ${ }^{35,36}$ Some of those reactions do not necessarily involve electron transfer. Details of the underlying mechanism for these prevailing phenomena, the power-law behavior, and the electronic states involved in single-particle fluorescence intermittency remain not fully understood.

Although an explanation can be given for the power-law intermittency based on a diffusion-reaction model, ${ }^{29,30}$ there are some important physical quantities that could not be extracted from these short-time data alone, such as the nature of the light and dark states and their energy gap. In powerlaw studies of single QDs, the blinking statistics $P(t)$ for either "light" or "dark" events was measured between $1 \mathrm{~ms}$ and $100 \mathrm{~s}$. Ensemble studies, in which fluorescence intensity $\langle I(t)\rangle$ can be measured at much longer times $\left(10^{4} \mathrm{~s}\right.$ or much longer ${ }^{23}$ ), can be used to obtain complementary information not readily accessible from single-QD studies.

Building upon our previous work that assumed a two-

\footnotetext{
${ }^{\text {a) }}$ Authors to whom correspondence should be addressed.

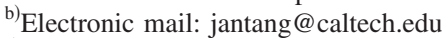

c)Electronic mail: ram@caltech.edu
}

state model,${ }^{31}$ we now include in this study a more elaborate model that includes the relevant band-edge exciton states, such as "light exciton," "dark exciton," and long-lived dark QD states and their roles in blinking. In this work we primarily focus on the normal diffusion case. Extension to anomalous diffusion ${ }^{29}$ could be made to address other power-law and ensemble behaviors. In our previous treatment, ${ }^{31}$ for simplicity we neglected the Stokes shift effects. These effects are not that small for some QDs. For example, as will be illustrated in this study of bare QDs without a ZnS shell, the Stokes shift is needed to provide a reasonably good fit to the data of Chung and Bawendi. ${ }^{23}$ As before, we also analyze existing data from different experiments on ensemble measurements and single-particle intermittency studies but now are able to extract Stokes shift and to compare it with the known value. ${ }^{8}$ With the improved model, we correct the values for the free energy gap between the light and dark states, the activation energy, and reorganization energy, so supplementing our earlier parameter list of the extracted quantities. ${ }^{31}$ As in our previous treatments, the electronic coupling is assumed to be weak so that the energy-level crossing between two parabolic potentials has a cusplike crossing, instead of a round-top crossing. A step toward addressing the round-top-crossing case will be explored in the Appendix.

\section{THE DIFFUSION-CONTROLLED ELECTRON TRANSFER MODEL AND THE RELEVANT ELECTRONIC STATES}

Under continuous illumination, each QD in an ensemble is assumed to involve slow electron transfer (blinking) processes between a pair of light and dark states. More specifically, the dark state $|D\rangle$ of Fig. 1(a) or $|2\rangle$ in Fig. 1(b) is considered to be a long-lived dark state which can last for hours or longer in the dark. ${ }^{23}$ Such a long lifetime indicates that $|2\rangle$ is weakly coupled electronically to the ground state $|0\rangle$ and to the light state $|1\rangle$. For example, $|2\rangle$ may be a triplet state, consisting of a positive core charge and a trapped sur- 
(a)

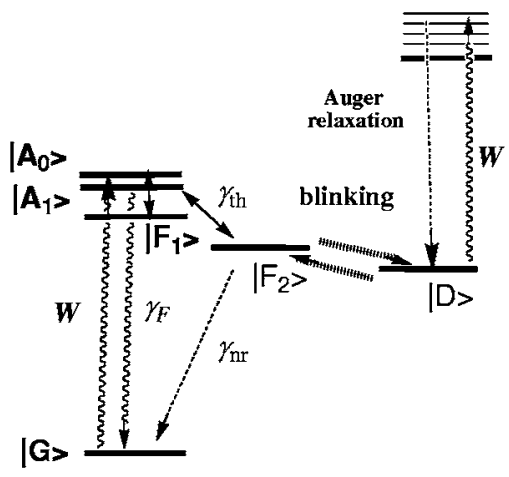

(b)

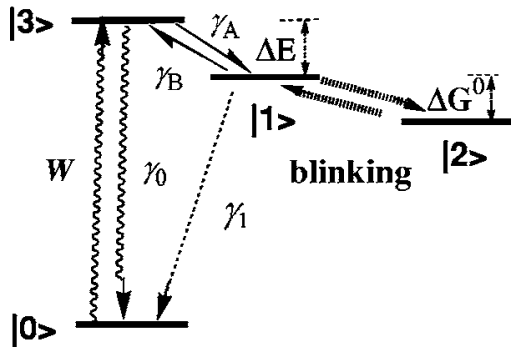

FIG. 1. (a) Schematic representation of the band-edge exciton structure of QDs. (b) Simplified scheme of relevant states for intermittency. The slow stochastic hopping between $|2\rangle$ and $|1\rangle$ represents the blinking processes. Initial population at the ground state $|0\rangle$ is first pumped by light to excited states (at $W \sim 10^{8} \mathrm{~s}^{-1}$ for $2 \mathrm{~kW} / \mathrm{cm}^{2}$ ), followed by rapid nonradiative relaxation $\left(\gg 10^{12} \mathrm{~s}^{-1}\right)$ to low-lying "light exciton" state $|3\rangle$ which establishes by thermal processes $\left(\gamma_{A} \sim 10^{10} \mathrm{~s}^{-1}\right)$ a quasiequilibrium with the "dark exciton" state $|1\rangle$. Decay $\gamma_{1}$ from $|1\rangle$ to $|0\rangle$ is nonradiative $\left(\sim 10^{6} \mathrm{~s}^{-1}\right)$ and fluorescence decay $\gamma_{0}$ from $|3\rangle$ to $|0\rangle$ occurs in $\sim 10^{8} \mathrm{~s}^{-1}$.

face state for the electron. In a different model by Frantsuzov and Marcus, ${ }^{32}$ deeply trap states with an Auger mechanism is assumed.

We consider $\left|F_{2}\right\rangle$ in Fig. 1(a) or $|1\rangle$ in Fig. 1(b) as a neutral dark exciton state, ${ }^{7-10}$ which has a total angular momentum projection $J$ of \pm 2 and is optically inactive..$^{7-10}$ There are several optically active states with similar oscillator strength for a radius of $25 \AA$, such as the lowest light exciton state $\left|F_{1}\right\rangle$ and two other higher lying states $\left|A_{1}\right\rangle$ and $\left|A_{0}\right\rangle{ }^{8,9}$ These band-edge exciton states $\left|A_{0}\right\rangle,\left|A_{1}\right\rangle,\left|F_{1}\right\rangle$, and $\left|F_{2}\right\rangle$ in Fig. 1(a) are denoted in Refs. 8 and 9 as $\left|0^{U}\right\rangle,\left| \pm 1^{U}\right\rangle$, $\left| \pm 1^{L}\right\rangle$, and $| \pm 2\rangle$, respectively. We use $|3\rangle$ in Fig. 1(b) to represent collectively all these optically active states. A rapid population recycling mechanism is induced by continuous photoexcitation $\left[W \sim 10^{8} \mathrm{~s}^{-1}\right.$ at $2 \mathrm{~kW} / \mathrm{cm}^{2}$ (Ref. 10) $]$ and the fast thermal process $\gamma_{\text {th }}$ (or $\gamma_{A}$ and $\gamma_{B}$ ) between $|3\rangle$ and $|1\rangle$ (Fig. 1). For a QD with a radius of $25 \AA$, the averaged gap $\Delta E$ between $|3\rangle$ and $|1\rangle$ is about $10 \mathrm{meV}$ (Refs. 8 and 9) and is dependent on size and shape. ${ }^{8-11}$ Although $|1\rangle\left(\right.$ or $\left.\left|F_{2}\right\rangle\right)$ is a dark exciton state in nature, the fast population recycling among $|1\rangle,|0\rangle$, and other optically active states $\left(\left|A_{1}\right\rangle,\left|A_{0}\right\rangle\right.$, and $\left.\left|F_{1}\right\rangle\right)$ makes this state appear light in the relevant time scale of usual intermittency measurements (greater than milliseconds). For these reasons, $|1\rangle$ is referred here as a light state.

In this model, diffusion is light driven and so electron transfer (ET) occurs even at cryogenic temperatures. For the charged dark state $|2\rangle$, due to fast Auger relaxation from the higher excited dark states (Fig. 1), its diffusion is slower and could have a light-independent component. ${ }^{23}$ In single-QD intermittency measurements, $P_{\text {on }}(t)$ or $P_{\text {off }}(t)$ represents the rate of change of the survival probability of a QD to remain in the light or dark state. In calculating $P_{\text {on }}(t)$ or $P_{\text {off }}(t)$, one only needs to consider either the forward or the reverse ET rate equation. However, $\langle I(t)\rangle$ represents overall light QDs in an ensemble in which each QD may have gone through numerous blinking cycles and is indistinguishable from the other. To describe $\langle I(t)\rangle$ of the ensemble-averaged fluorescence decay, one needs to use coupled rate equations including both forward and reverse transitions. We consider the following equations for populations among four states $(|0\rangle$, $|1\rangle,|2\rangle$, and $|3\rangle)$ in Fig. 1(b), where the reaction between $|1\rangle$ and $|2\rangle$ is diffusion controlled. The following rate equations for the four states in Fig. 1(b) are an extension to the previous two-state equations: ${ }^{31}$

$$
\begin{aligned}
\frac{\partial}{\partial t} \rho_{0}(Q, t) & =\gamma_{0} \rho_{3}(Q, t)+\gamma_{1} \rho_{1}(Q, t)-W \rho_{0}(Q, t), \\
\frac{\partial}{\partial t} \rho_{3}(Q, t)= & \gamma_{B} \rho_{1}(Q, t)+W \rho_{0}(Q, t)-\left(\gamma_{A}+\gamma_{0}\right) \rho_{3}(Q, t), \\
\frac{\partial}{\partial t} \rho_{1}(Q, t)= & \gamma_{A} \rho_{3}(Q, t)-\left(\gamma_{B}+\gamma_{1}\right) \rho_{1}(Q, t)+L_{1} \rho_{1}(Q, t) \\
& -\frac{2 \pi\left|V_{\mathrm{ex}}\right|^{2}}{\hbar} \delta\left(U_{12}(Q)\right)\left(\rho_{1}(Q, t)-\rho_{2}(Q, t)\right),
\end{aligned}
$$

$$
\begin{aligned}
\frac{\partial}{\partial t} \rho_{2}(Q, t)= & L_{2} \rho_{2}(Q, t)-\frac{2 \pi\left|V_{\mathrm{ex}}\right|^{2}}{\hbar} \delta\left(U_{12}(Q)\right)\left(\rho_{2}(Q, t)\right. \\
& \left.-\rho_{1}(Q, t)\right) .
\end{aligned}
$$

Assuming a slow ET rate and weak electronic coupling, one can solve and simplify the first three equations in Eq. (1) by Laplace transformation. Defining $s$ as a variable in the Laplace transform domain, $\bar{\rho}_{0}(s)$ and $\bar{\rho}_{3}(s)$ can be expressed in terms of $\bar{\rho}_{1}(s)$,

$$
\begin{aligned}
& {\left[s+\gamma_{1}+\gamma_{B}-\frac{\gamma_{A}\left(\gamma_{B}(s+W)+W \gamma_{1}\right)}{s\left(W+\gamma_{0}+\gamma_{A}\right)+W \gamma_{A}}\right] \bar{\rho}_{1}(Q, s)} \\
& =L_{1} \bar{\rho}_{1}(Q, s)-\frac{2 \pi\left|V_{\mathrm{ex}}\right|^{2}}{\hbar} \delta\left(Q-Q_{c}\right)\left(\bar{\rho}_{1}(Q, s)-\bar{\rho}_{2}(Q, s)\right) \\
& \quad+\rho_{1, \mathrm{eff}}(Q, 0) \\
& s \bar{\rho}_{2}(Q, s)=L_{2} \bar{\rho}_{2}(Q, s)-\frac{2 \pi\left|V_{\mathrm{ex}}\right|^{2}}{\hbar} \delta\left(Q-Q_{c}\right)\left(\bar{\rho}_{2}(Q, s)\right. \\
& \left.\quad-\bar{\rho}_{1}(Q, s)\right),
\end{aligned}
$$

where $L_{k}$ is the diffusion operator $L_{k} \equiv\left(\Delta^{2} / \tau_{L, k}\right)(\partial / \partial Q)$ $\times\left(\partial / \partial Q+\partial U_{k}(Q) / k_{B} T \partial Q\right)$. In this study we consider a blinking process (diffusion reaction between $|1\rangle$ and $|2\rangle$ ) much slower than other rate processes. Assuming a fast population recycling among $|0\rangle,|3\rangle$, and $|1\rangle$, Eq. (2) can be reduced 
approximately to a two-state rate equation involving only $\bar{\rho}_{1}(s)$ and $\bar{\rho}_{2}(s)$ as

$$
\begin{aligned}
s \bar{\rho}_{1}(Q, s)-\rho_{1}(Q, 0) \approx & L_{1, \mathrm{eff}} \bar{\rho}_{1}(Q, s)-A_{1} \delta\left(Q-Q_{c}\right) \\
& \times\left(\bar{\rho}_{1}(Q, s)-\bar{\rho}_{2}(Q, s)\right), \\
s \bar{\rho}_{2}(Q, s)= & L_{2, \mathrm{eff}} \bar{\rho}_{2}(Q, s)-A_{2} \delta\left(Q-Q_{c}\right)\left(\bar{\rho}_{2}(Q, s)\right. \\
& \left.-\bar{\rho}_{1}(Q, s)\right) .
\end{aligned}
$$

Here, we define $L_{k, \text { eff }} \equiv \varsigma_{k} L_{k}, \quad\left|V_{k}\right|^{2} \equiv \varsigma_{k}\left|V_{\text {ex }}\right|^{2}, \quad \varsigma_{1}$ $\equiv W \gamma_{A} /\left(W\left(\gamma_{A}+\gamma_{B}+\gamma_{1}\right)+\gamma_{0} \gamma_{B}+\gamma_{1} \gamma_{0}+\gamma_{1} \gamma_{A}\right), \quad \varsigma_{2} \equiv 1, \quad V_{\mathrm{ex}}$ the electronic coupling, $\quad A_{k} \equiv \varsigma_{k}(2 \pi / \hbar)\left|V_{\text {ex }}\right|^{2} /$ $\times\left|\partial\left(U_{12}(Q)\right) / \partial Q\right|_{Q=Q_{c}}$, and $1 / \tau_{k} \equiv \varsigma_{k} / \tau_{L, k}$. A fast population recycling among $|0\rangle,|3\rangle$, and $|1\rangle$ has thus led to an effective population $\varsigma_{1}$ for $|1\rangle$ at $t \gg 10^{-6} \mathrm{~s}\left(1 / \gamma_{1}\right.$ and $\left.1 / W\right)$. In this diffusion-controlled electron transfer (DCET) model, diffusion in energy space on two parabolic potentials (Fig. 2) is light driven. $Q_{c}$ is the energy-level crossing point of two potentials with $U_{12}\left(Q_{c}\right)=0$, where $U_{1}(Q)=\kappa\left(Q-Q_{0,1}\right)^{2} / 2$, $U_{2}(Q)=\kappa\left(Q-Q_{0,2}\right)^{2} / 2+\Delta G^{0}, \quad \kappa$ the force constant with $\kappa \Delta^{2}=k_{B} T$ at room temperature, $\Delta G^{0}$ the free energy gap, and $\lambda=\kappa\left(Q_{0,1}-Q_{0,2}\right)^{2} / 2$ the reorganization energy.

We consider here normal diffusion which has been shown to yield $t^{-3 / 2}$ power-law intermittency, ${ }^{29}$ a value close to the observations by Chung and Bawendi, ${ }^{23}$ whose pioneering work is the focus of the present treatment. In their ensemble measurements ${ }^{23}$ of $\langle I(t)\rangle$ QDs are initially in darkness, presumably in the ground state. When light illumination was stopped after each cycle of $\langle I(t)\rangle$ measurements, the intensity recovered its full intensity after a long period $(8000 \mathrm{~s})$ until starting the next measurement cycle. Thus, we assume that QDs are in $|0\rangle$ initially in thermal equilibrium prior to each cycle of light illumination. Due to fast population recycling described above and the presence of a Stokes shift ${ }^{8}$ between $|3\rangle$ and $|1\rangle$, the excited population distribution is centered at $Q_{0,3}$ rather than at $Q_{0,1}$, the lowest point of $U_{1}(Q)$. Here, an initial distribution of QDs in $|1\rangle$ is assumed to have a Stokes shift as shown in Fig. 2(b), after the initial transient period, $\rho_{1}(Q, 0)=\left(1 / \sqrt{2 \pi k_{B} T / \kappa}\right)$ (a)

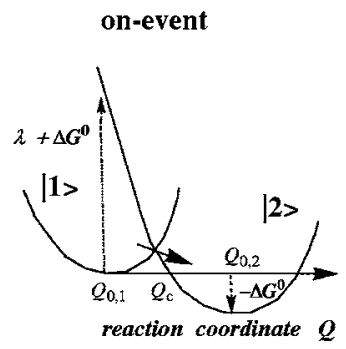
off-event

(b)
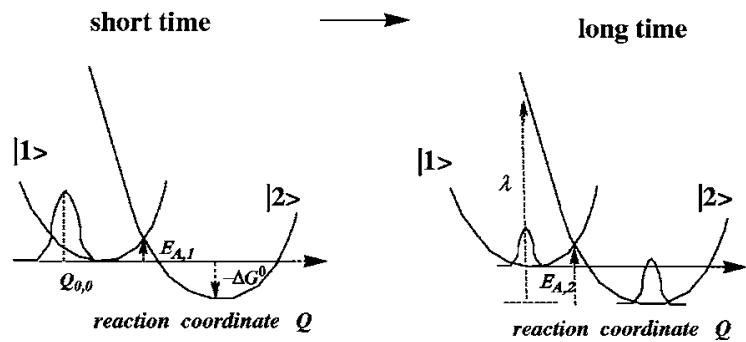

FIG. 2. (a) Diffusion on the parabolic potential surfaces for $|1\rangle$ and $|2\rangle$ across a sink $Q_{c}$ at the energy-level crossing governs the intermittency phenomenon. (b) Time evolution of an initial population profile centered at $Q_{0,0}$ in $|1\rangle$ and a steady-state distribution at long times between $|1\rangle$ and $|2\rangle$. Relation of forward/reverse ET activation energy $\left(E_{A, 1} / E_{A, 2}\right)$, reorganization energy $(\lambda)$, and free energy gap $\left(\Delta G^{0}\right)$ to two parabolas is illustrated.

$\times \exp \left(-\kappa\left(Q-Q_{0,0}\right)^{2} / 2 k_{B} T\right)$, where $Q_{0,0}$ is the center position for the distribution. The normalized intensity $\langle\bar{I}(s)\rangle$ can be derived from Eq. (3),

$$
\begin{aligned}
\langle\bar{I}(s)\rangle \equiv & \int_{-\infty}^{\infty} d Q \overline{\rho_{1}}(Q, s)=\frac{1}{s} \int_{-\infty}^{\infty} d Q \rho_{1}(Q, 0) \\
& -\frac{\left.\int_{-\infty}^{\infty} d Q A_{1} \bar{G}_{1}\left(Q_{c}, Q ; s\right) \rho_{1} Q, 0\right)}{s\left(1+A_{1} \bar{G}_{1}\left(Q_{c}, Q_{c} ; s\right)+A_{2} \bar{G}_{2}\left(Q_{c}, Q_{c} ; s\right)\right)},
\end{aligned}
$$

where Green's function $\bar{G}_{k}\left(Q, Q^{\prime} ; s\right)$ satisfies $s \bar{G}_{k}\left(Q, Q^{\prime} ; s\right)$ $-L_{k, \text { eff }} \bar{G}_{k}\left(Q, Q^{\prime} ; s\right)=\delta\left(Q-Q^{\prime}\right)$. One has more specifically

$$
\langle\bar{I}(s)\rangle=\frac{1}{s}-\frac{\gamma_{1} \int_{0}^{\infty} d t \exp \left(-s t-2 \Gamma_{1} \tau_{1}\left[\left(1+\xi \exp \left(-t / \tau_{1}\right)^{2}-1\right)\right]\right)}{s\left(1+A_{1} \bar{G}_{1}\left(Q_{c}, Q_{c} ; s\right)+A_{2} \bar{G}_{2}\left(Q_{c}, Q_{c} ; s\right)\right)},
$$

where $\xi \equiv\left(Q_{0,1}-Q_{0,0}\right) /\left(Q_{c}-Q_{0,1}\right)$ and the Stokes shift $\Lambda$ $=\xi^{2} E_{A, 1} / 2$. The steady-state intensity $I_{\text {eq }}$ is given by

$$
I_{\mathrm{eq}}=\lim _{s \rightarrow 0} s \bar{\rho}_{1}(s)=\frac{\varsigma_{2} \gamma_{2}}{\varsigma_{1} \gamma_{1}+\varsigma_{2} \gamma_{2}}=\frac{1}{1+\varsigma_{1} e^{-\Delta G^{0} / k_{B} T}},
$$

where $\quad \lim _{s \rightarrow 0} s \bar{g}_{k}(s)=\varsigma_{k} \gamma_{k}, \quad \varsigma_{k} \gamma_{k} \equiv\left(A_{k} / \sqrt{2 \pi \Delta_{k}^{2}}\right) \exp \left(-E_{A, k} /\right.$ $\left.k_{B} T\right), \gamma_{1}$ and $\gamma_{2}$ are the nonadiabatic forward and reverse ET reaction rates with activation energies $E_{A, 1}=\left(\lambda+\Delta G^{0}\right)^{2} / 4 \lambda$ and $E_{A, 2}=\left(\lambda-\Delta G^{0}\right)^{2} / 4 \lambda$, and

$$
\Gamma_{k} \tau_{k}=E_{A, k} / 2 k_{B} T, \quad 2 \Gamma_{1} \tau_{1}-2 \Gamma_{2} \tau_{2}=\Delta G^{0} / k_{B} T .
$$

The relation of $E_{A, 1}, E_{A, 2}, \lambda$, and $\Delta G^{0}$ to the parabolic potentials is illustrated in Fig. 2.

When the electronic coupling is sufficiently large and the effective potential near the energy-level crossing has a round 
top as illustrated in Fig. 3(a), in such a regime the exact analytic Green's function solution for such an effective potential in the DCET model is not available, and a numerical approach has to be employed. A step toward treating this problem is given in the Appendix.

\section{DATA ANALYSIS AND DISCUSSION}

In this section, we analyze the existing data from several experiments by Bawendi's group to extract a number of molecular-based parameters.

To evaluate $\langle I(t)\rangle$ from Eq. (5), a standard numerical inverse Laplace transform method was used and was described previously. ${ }^{31}$ More expansion terms may be needed for a larger $\xi$ or $\Gamma_{k}$. Some calculated curves for $\langle I(t)\rangle$ are illustrated in Fig. 4 to show how kinetic and energetic variables control the temporal behavior of $\langle I(t)\rangle$.

Using Eq. (5) we analyzed and fitted the experimental data for CdSe QDs, taken from Fig. 3(c) of Ref. 23, measured at room temperature and at about $2 \mathrm{~kW} / \mathrm{cm}^{2}$. Based on the photoabsorption cross section for QDs with an $18 \AA$ radius $^{37}$ and extrapolated to $25 \AA$, one has $W \sim 1.1$ $\times 10^{8} \mathrm{~s}^{-1}$. With $k_{B} T \sim 25 \mathrm{meV}, \Delta E \sim 10 \mathrm{meV}, 8,9 \gamma_{A} / \gamma_{B}$ $\sim 1.5,{ }^{10} \gamma_{A} \sim 10^{10} \mathrm{~s}^{-1}, \quad \gamma_{0} \sim 5 \times 10^{7} \mathrm{~s}^{-1}$, and $\gamma_{1} \sim 10^{6} \mathrm{~s}^{-1},{ }^{10}$ one has $s_{1} \sim 0.5$. From the analysis of the $\langle I(t)\rangle$ data and the fitted $\Gamma_{1}$ from $P_{\text {on }}(t)$, taken from Fig. 2(a) of Ref. 23, some useful parameters can be extracted for the CdSe sample [Fig. 5(a)] and the other CdSe sample with a $\mathrm{ZnS}$ shell [Fig. 5(b)]. A list of the extracted physical parameters is given in Table I. $^{38}$ The Stokes shift, defined as the energy difference between the peak of band-edge absorption and the peak of full luminescence according to a study by Efros et al., is about 40-50 meV for QDs with a radius of 20-30 $\AA$ and is comparable to the value extracted from $\langle I(t)\rangle$. A similar table of parameters was shown in our earlier work, ${ }^{31}$ but those parameters were estimated for CdSe QDs with a $\mathrm{ZnS}$ shell only assuming the Stokes shift is negligible. The table of this study also contains parameters for CdSe without a shell. Here, an experimental value for $1 / \Gamma_{1}$ from the observed bending tail was used and the band-edge exciton states of Figs. 1(a) and 1(b) were taken into consideration. Because diffusion and energetics are sensitive to experimental conditions, more accurate determination of a consistent set of these parameters requires systematic measurements of QDs under the same conditions of light intensity, temperature, size, and material composition. At present, the measurements were performed at different conditions and on different samples rather than all on the same system.

In addition to spectral diffusion induced by energy fluctuation, ${ }^{9}$ slow variations in fluorescence intensity level of the "on" periods and the decay time to the dark state were reported. ${ }^{25}$ Such variations could result from fluctuations in the energy gap $\Delta E$ between $|1\rangle$ and $\left|F_{1}\right\rangle,\left|A_{1}\right\rangle$, or $\left|A_{0}\right\rangle$ (Fig. $1)$. Because the quasiequilibrium population ratio depends on $\Delta E$, when $\Delta E$ fluctuates the fluorescence intensity of the on level and the transition time constant can also change. In our earlier work, ${ }^{29}$ two regimes, rather than one, of a power law were predicted. At times much shorter than the time constant $t_{c}$, one has a $t^{-1 / 2}$ regime that changes to a $t^{-3 / 2}$ regime at (a)

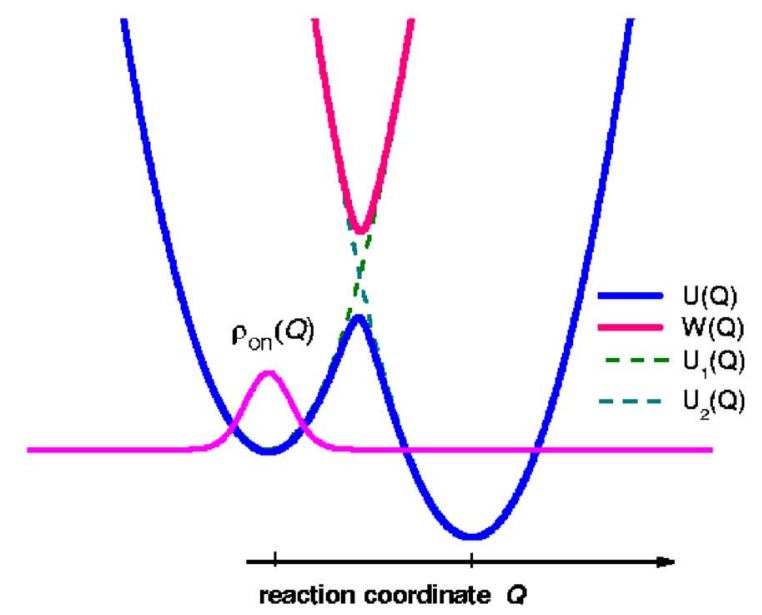

(b)

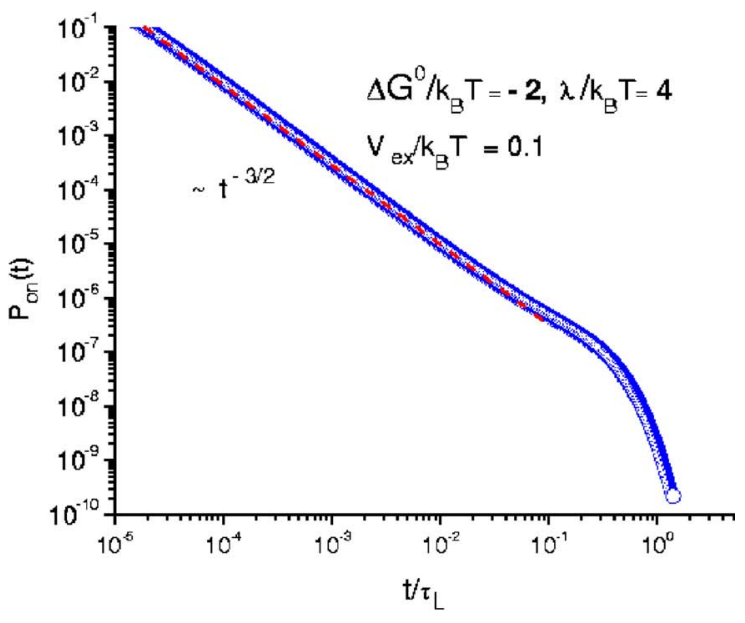

(c)

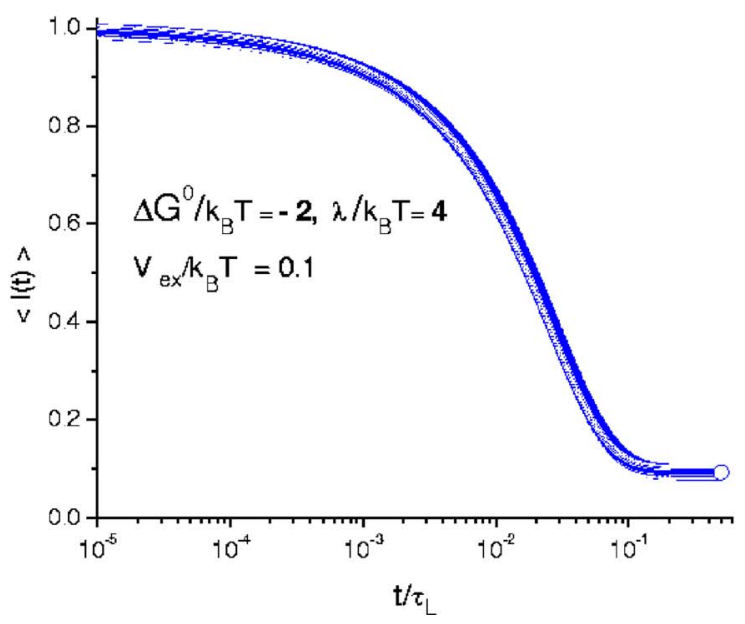

FIG. 3. (a) Effective potential energy surfaces $W(Q)$ and $U(Q)$ for $|1\rangle$ and $|2\rangle$ when the electronic coupling is net negligibly small. The lower potential $W(Q)$ near the crossing has a round top and the electron transfer reactions become a Kramers-type reaction. (b) The "on" event blinking statistics $P_{\text {on }}(t)$ showing a $t^{-3 / 2}$ power law and an exponential tail at long times. The time unit for the $x$ axis is $1 / \tau_{L}$. (c) Semilog plot of $\langle I(t)\rangle$ which approaches an equilibrium value $I_{\text {eq }}$ at longer times. In the simulations, $\Delta G^{0} / k_{B} T=-2$, $\lambda / k_{B} T=4$, and $V_{\mathrm{ex}} / k_{B} T=0.1$ were used with 1000 discrete points over a span of 10 for $q$. 
(a)

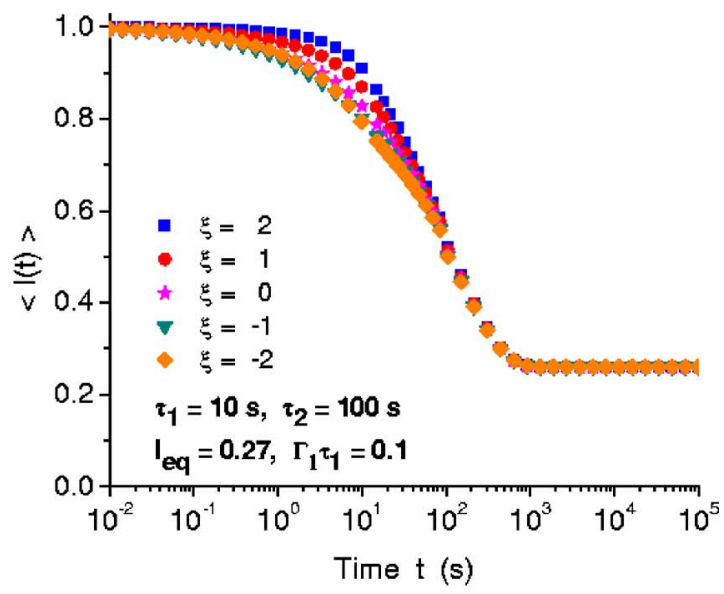

(b)

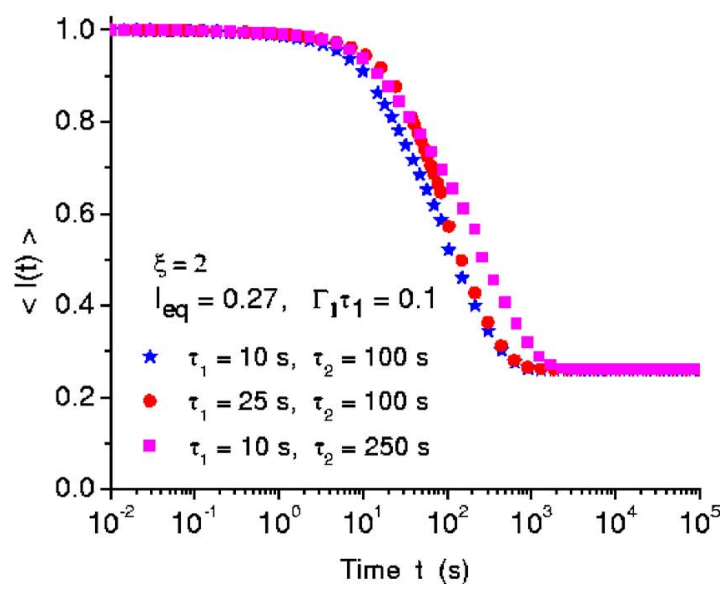

FIG. 4. (a) Semilog plot of $\langle I(t)\rangle$ for various Stokes shift parameter $\xi$. A large positive $\xi$ represents an initial population profile further away from the energy-level crossing point. It causes the bending of $\langle I(t)\rangle$ from the plateau to start later in time. (b) Semilog plot of $\langle I(t)\rangle$ for various diffusion correlation times $\tau_{1}$ and $\tau_{2}$. These parameters control the bending time of $\langle I(t)\rangle$ from the initial plateau and the relaxation time for $\langle I(t)\rangle$ to approach an equilibrium value $I_{\text {eq. }}$

times much greater than $t_{c}$. The value of $t_{c}$ is presently unknown. The fitting of experimental data in Fig. 5 is not sensitive to the choice of $t_{c}$ as long as $t_{c} \gg 100 \mathrm{~s}$. If one picks a $V_{\text {ex }}$ on the order of $100 \mathrm{meV}$, using $t_{c, k}$ $\equiv 2 \lambda k_{B} T \hbar^{2} / \pi^{2} \tau_{k}\left|V_{k}\right|^{4}$ from our earlier work, ${ }^{31}$ one can show that the inequality relation for $t_{c}$ is satisfied with such a $V_{\mathrm{ex}}$ which corresponds to a $t_{c} \ll 1 \mathrm{fs}$. Therefore, the $t^{-1 / 2}$ regime for $t \ll t_{c}$ is unobservable. The value for $V_{\mathrm{ex}}$ cannot be determined from our analysis of the data of Chung and Bawendi. ${ }^{23}$ Its determination and the question about the distance between the electron and hole in the trap state have to rely on other means that remain to be explored. The cornerstone of our model is the diffusion-controlled reaction, and the $t^{-3 / 2}$ power-law behavior in fluorescence intermittency of single QDs is a consequence of slow diffusion in energy space.

The following consequences are expected for the DCET model and await further direct experimental tests. (a)

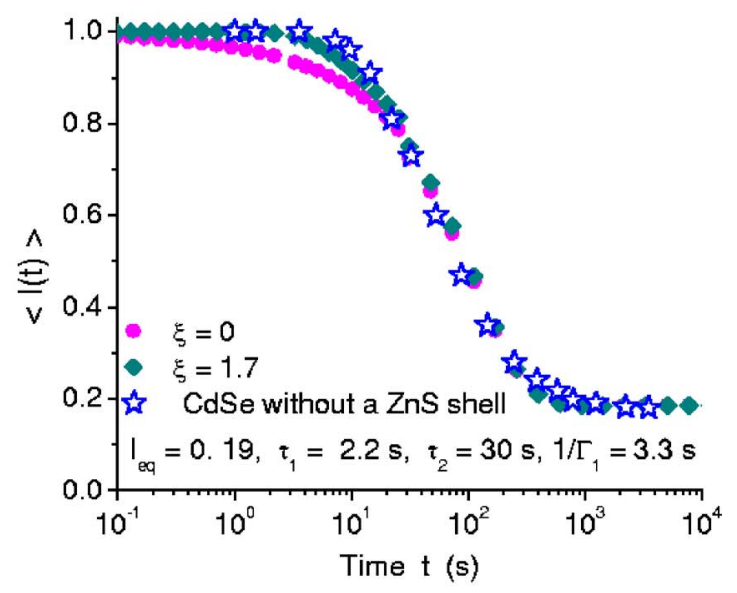

(b)

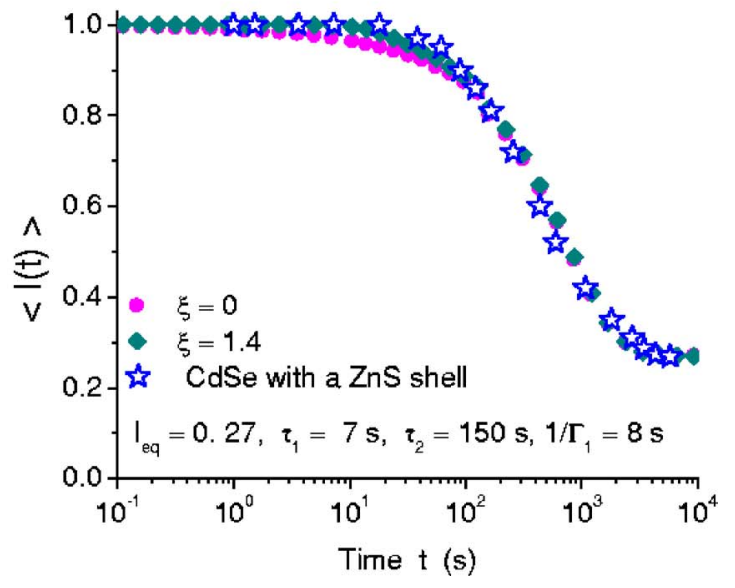

FIG. 5. (a) Semilog plot of experimental $\langle I(t)\rangle$ vs the fitted curve. CdSe QDs with no shell. (b) Experimental $\langle I(t)\rangle$ vs the fitted curve for CdSe QDs with a $\mathrm{ZnS}$ shell.

(1) According to Eq. (7), the bending rate $\Gamma_{k}$ is predicted to increase linearly with light intensity $W$. Because the diffusion is light induced for the light QDs, according to the observation of Empedocles and Bawendi, ${ }^{16} 1 / \tau_{k}$

TABLE I. A list of estimated physical parameters.

\begin{tabular}{|c|c|c|}
\hline Parameters & $\mathrm{CdSe}$ & $\mathrm{CdSe}(\mathrm{ZnS})$ \\
\hline $1 / \Gamma_{1}(\mathrm{~s})$ & 3.3 & 8 \\
\hline$\Delta G^{0}$ (Free energy gap) (meV) & -53 & -41 \\
\hline$\Lambda$ (Stokes shift) (meV) & 48 & 43 \\
\hline$E_{A, 1}$ ("light-to-dark" activation energy) (meV) & 33 & 44 \\
\hline$E_{A, 2}$ ("dark-to-light" activation energy) (meV) & 86 & 85 \\
\hline$\lambda(\text { reorganization energy })^{\mathrm{a}}(\mathrm{meV})$ & 226 or 12 & 250 or 7 \\
\hline $\begin{array}{c}\tau_{1} \\
\text { (light-state diffusion correlation time) }^{\mathrm{b}}(\mathrm{s})\end{array}$ & 2 & 7 \\
\hline $\begin{array}{c}\tau_{2} \\
\text { (dark-state diffusion correlation time) (s) }\end{array}$ & 35 & 150 \\
\hline
\end{tabular}

$\overline{\mathrm{a}}$ Two possible solutions exist for $\lambda$ from a quadratic equation, corresponding to the "normal" and "inverted" regimes.

${ }^{\mathrm{b}}$ This time at room temperature is much shorter than the times obtained at $10 \mathrm{~K}-40 \mathrm{~K}$ from spectral diffusion data (given in Table I of Ref. 30). 
is linearly proportional to the light intensity $W$. At much higher intensity, however, saturation and generation of multiexcitons can complicate the linear dependence. The dependence of diffusion for QDs in the dark is less studied and may have a weaker dependence.

(2) The breakdown of the power law for $P_{\text {off }}(t)$ would occur as $P_{\text {on }}(t)$, but at a later time than $P_{\text {on }}(t)$. At very long times the power law for both on and "off" events should change to an exponential decay. According to Table I a bending in $P_{\text {off }}(t)$ for CdSe of $25 \AA$ is expected to occur by $100 \mathrm{~s}$ at room temperature, at a light intensity of $2 \mathrm{~kW} / \mathrm{cm}^{2}$. A bending for $P_{\text {off }}(t)$ has not been reported, but its existence is supported by the observation that $\langle I(t)\rangle$ reaches a steady state at about $10^{3} \mathrm{~s}$ (Fig. 5).

(3) The ratio of steady-state fluorescence intensity and the intensity after the initial transient period of an ensemble system is given by $I_{\mathrm{eq}}=1 /\left(1+\varsigma_{1} e^{-\Delta G^{0} / k_{B} T}\right)$. This ratio depends on light intensity $W$ through the population factor $s_{1} \equiv W \gamma_{A} /\left(W\left(\gamma_{A}+\gamma_{B}+\gamma_{1}\right)+\gamma_{0} \gamma_{B}+\gamma_{1} \gamma_{0}+\gamma_{1} \gamma_{A}\right)$, and so a reduction in light intensity should make $I_{\text {eq }}$ increase. At a much higher light intensity, due to complication of possible multiexciton generation, $\langle I(t)\rangle$ may differ from the quasistretched exponential behavior exhibited in Fig. 4.

This study and our previous reports ${ }^{29-31}$ are based on a model with photoinduced spectral diffusion and diffusioncontrolled electron transfer for QDs. Several of the consequences of the DCET model can be put to further tests.

(1) According to our previous analysis of spectral diffusion data, $^{29}$ the second moment follows $\sigma^{2}(t) / \sigma^{2}(0)=1$ $-\exp \left(-t / \tau_{1}\right)$. Because $1 / \tau_{k}$ is linearly proportional to the light intensity $W$, a similar dependence of $\sigma^{2}(t)$ is expected on a universal variable of energy density, i.e., $W t$, in accordance with the earlier experiments. ${ }^{16}$

(2) The power-law behavior for $P_{\text {on }}(t)$, like the spectral diffusion, should also follow a similar power-law dependence on an universal variable of energy density at lower light intensity. At a much higher intensity, saturation and multiexciton can complicate such a universal dependence.

(3) For QDs with negligible Stokes shift, treated previously, ${ }^{31}$ a universal dependence is expected for the exponent and time constant on several kinetic and energetic parameters in the stretched exponential decay fit to ensemble-averaged fluorescence intensity. Such a simple universal dependence is expected to break down when Stokes shift effects are included. Further studies are required to examine how much the universal relationship is affected.

\section{CONCLUSION}

As illustrated in the present study and our earlier work, ${ }^{29-31}$ the diffusion-controlled electron transfer model is shown to yield power-law intermittency for single QDs and a quasistretched exponential decay for ensemble-averaged fluorescence decay. Both single-particle and ensemble measurements are needed to provide a more complete picture for the underlying mechanism, and these data were analyzed to extract physical parameters. By including a Stokes shift effect in the DCET model, we obtained better fits to both sets of experimental data for CdSe QDs with and without a $\mathrm{ZnS}$ shell. Without the Stokes shift effect, i.e., $\xi=0$, the fit was poor, as illustrated in Fig. 5(a). The roles of various bandedge exciton states and a long-lived dark state in intermittency are included, and a molecular-based model serves to link the ensemble-averaged fluorescence intensity decays to single QDs intermittency. In this study, we focused on the data analysis of CdSe QDs. These samples ${ }^{23}$ show a powerlaw intermittency with an exponent close to $-3 / 2$, an ideal case where complications due to anomalous diffusion can be neglected.

Although the present work focuses on the normal diffusion case that leads to a power law with an exponent of $-3 / 2$, the anomalous diffusion case with a different exponent has been studied previously. ${ }^{29}$ The extension of Eq. (1) to the anomalous diffusion can be made by replacing the diffusion operator $L_{k}$ by an operator involving a time-dependent diffusion constant. The inclusion of anomalous diffusion changes the exponent of the power-law blinking in single QDs and also affects the exponent of the quasistretched exponential decay for the ensemble fluorescence intensity. The details have been addressed in another work. ${ }^{39}$

The blinking phenomenon in single quantum dots has also been observed in enzyme activity of single biomolecules $^{33,34}$ and other single organic molecules. ${ }^{35,36}$ This activity could be modeled as Kramers-type potential as illustrated in the Appendix. However, the details about diffusion-controlled reactions in these systems remain to be explored.

We have determined relevant parameters such as the free energy gap the Stokes shift, the activation energy for both "light-to-dark" and "dark-to-light" reactions, the reorganization energy, and other kinetic parameters. One key entity remains unspecified is the electronic coupling $V_{\mathrm{ex}}$ which needs to be determined by other means. Quantification of the molecular-based parameters improves the understanding of the underlying mechanism and the roles of band-edge exciton structure in intermittency of single QDs and ensembleaveraged fluorescence decay.

\section{ACKNOWLEDGMENTS}

The authors acknowledge the support of the National Science Foundation and the Office of Naval Research. One of the authors (J.T.) also thanks the James W. Glanville Foundation at the California Institute of California for the support. The same author (J.T.) benefited from discussions with Chung and Bawendi about their experiments that are central to this study. 


\section{APPENDIX: TREATMENT OF THE DCET MODEL INVOLVING AN EFFECTIVE POTENTIAL WITH A ROUND TOP NEAR THE CROSSING}

The effective potential $W(Q)$, as illustrated in Fig. 3(a), is due to mixing of two harmonic potentials $U_{1}(Q)$ and $U_{2}(Q)$ by the electronic coupling $V_{\mathrm{ex}}$, where $U_{1}(Q)=\kappa(Q$ $\left.+Q_{0}\right)^{2} / 2, U_{2}(Q)=\kappa\left(Q-Q_{0}\right)^{2} / 2+\Delta G^{0}, \kappa$ the force constant with $\kappa \Delta^{2}=k_{B} T$ at room temperature, $\Delta G^{0}$ the free energy gap, and $\lambda=2 \kappa Q_{0}^{2}$ the reorganization energy. Due to such a mixing, two effective potentials, the lower potential $W(Q)$ and the upper potential $U(Q)$, are obtained,

$$
\begin{aligned}
& W(Q)=\frac{U_{1}(Q)+U_{2}(Q)}{2}-\sqrt{\left(\frac{U_{1}(Q)-U_{2}(Q)}{2}\right)^{2}+V_{\mathrm{ex}}^{2}}, \\
& U(Q)=\frac{U_{1}(Q)+U_{2}(Q)}{2}+\sqrt{\left(\frac{U_{1}(Q)-U_{2}(Q)}{2}\right)^{2}+V_{\mathrm{ex}}^{2}} .
\end{aligned}
$$

The reaction coordinate $Q_{c}$ for the round top near the energylevel crossing is defined as $\partial W(Q) / \partial Q=0$ at $Q=Q_{c}$, and $Q_{c}$ can be approximated by

$$
Q_{c} \approx \frac{Q_{0} \Delta G^{0}}{\lambda-\sqrt{4 V_{\mathrm{ex}}^{2}+\left(U_{1}-U_{2}\right)^{2}\left(\frac{Q_{0} \Delta G^{0}}{\lambda-2 V_{\mathrm{ex}}}\right)^{2}}} .
$$

The diffusion equation involving such an effective potential $W(Q)$ can be written as

$$
\frac{\partial}{\partial t} \rho(Q, t)=\frac{\Delta^{2}}{\tau_{L}} \frac{\partial}{\partial Q}\left[\frac{\partial}{\partial Q}+\frac{1}{k_{B} T} \frac{\partial W(Q)}{\partial Q}\right] \rho(Q, t) .
$$

By defining a dimensionless variable $q$ for the reaction coordinate with $q=Q / \Delta$ and a dimensionless potential $w(q)$ $=W(Q) / k_{B} T$, one can express Eq. (A3) as

$$
\begin{aligned}
\frac{\partial}{\partial t} \rho(q, t)= & \frac{1}{\tau_{L}} \frac{\partial^{2}}{\partial q^{2}} \rho(q, t)+\frac{\rho(q, t)}{\tau_{L}} \frac{\partial^{2}}{\partial q^{2}} w(q) \\
& +\frac{1}{\tau_{L}} \frac{\partial w(q)}{\partial q} \frac{\partial}{\partial q} \rho(q, t) .
\end{aligned}
$$

The diffusion-controlled reaction in the lower W-shaped potential $W(Q)$ is essentially equivalent to the Kramers reaction. ${ }^{40}$ Because $w(q)$ is no longer a harmonic potential, Green's function for the above diffusion equation cannot be obtained in a simple analytic form. A numerical approach using the finite element method with sufficient lattice points can be used to solve the diffusion equation.

If the mixing due to the electronic coupling is strong, the states near the round top actually represent a quantum mixture between the light and the dark states. Subtleties as to how to clearly define a light or dark state around the top will not be treated here, but any treatment would note that the usual bin time $\left(10^{-2} \mathrm{~s}\right.$ or greater $)$ is large. For simplicity, we neglect the possibility of recrossing to the upper states; we simply define the light state (or the dark state) to the left (or right) of the round top of the $\mathrm{W}$-shaped potential. One can then define the single-particle blinking statistics $P_{\text {on }}(t)$ and $P_{\text {off }}(t)$ as

$$
P_{\mathrm{on}}(t) \equiv-\frac{d}{d t} \int_{-\infty}^{q_{c}} d q \rho(q, t), \quad P_{\mathrm{off}}(t) \equiv-\frac{d}{d t} \int_{q_{c}}^{\infty} d q \rho(q, t),
$$

and an initial population $\delta\left(q-q_{c}\right)$ is assumed at the round top $q_{c}=Q_{c} / \Delta$ defined in Eq. (A2). The blinking statistics $P(t)$ is equivalent to the probability flux at the round top of the Kramers potential. In addition, due to a priori information in single QDs as explained in our previous work, ${ }^{30}$ to treat blinking statistics of single QDs the usual Zusman rate equations need to be decoupled, i.e., for $P_{\text {on }}(t)$ there is no need to include the reverse electron transfer from the dark state back to the light state. The transitions around the round top between the upper and lower states due to quantum mixing are neglected. Similarly, in treating the Kramers-type reaction, to guarantee no reverse reaction one can add a fast decay term to those populations in the dark states (in the right half of the W-shaped potential). Some simulated results for $P_{\text {on }}(t)$ with various energetic and coupling parameters are illustrated in Fig. 3(b), showing the $t^{-3 / 2}$ power law as also obtained for the DCET model with two harmonic potentials in the previous studies. ${ }^{30}$ The major difference is that the $t^{-1 / 2}$ regime at very short time in the previous DCET model is absent here.

To calculate the time evolution of the fluorescence intensity $\langle I(t)\rangle$ for the light state, one solves Eq. (A3) or (A4) with an initial Boltzmann population in the light state. The simulated results using the sixth order Runge-Kutta method with discrete lattice points in $q$ are illustrated in Fig. 3(c), showing an equilibrium intensity reached at times much longer than $\tau_{L}$. These results for Kramers-type reaction are similar to those obtained previously by the DCET model. ${ }^{31}$

${ }^{1}$ L. Jacak, P. Hawrylak, and A. Wojs, Quantum Dots (Springer, Berlin, 1997).

${ }^{2}$ Quantum Dots: A Doorway to Nanoscale Physics, edited by W. D. Heiss (Springer, Berlin, 2005).

${ }^{3}$ Nanoparticles: Building Blocks for Nanotechnology, edited by V. Botello (Kluwer Academic, New York, 2004).

${ }^{4}$ W. Min, B. P. English, B. J. Cheravil, S. C. Kou, and X. S. Xie, Acc. Chem. Res. 38, 923 (2005); G. Bokinsky and X. Zuang, Acc. Chem. Res. 38, 566 (2005).

${ }^{5}$ Single Molecule Spectroscopy, edited by R. Rigler, M. Orrit, and T. Basché (Springer-Verlag, Heidelberg, 2002).

${ }^{6}$ W. J. Parak, T. Pellegrino, and C. Plank, Nanotechnology 16, R9 (2005). ${ }^{7}$ M. Nirmal, D. J. Norris, M. Kuno, M. G. Bawendi, A. L. Efros, and M. Rosen, Phys. Rev. Lett. 75, 3728 (1995).

${ }^{8}$ A. L. Efros, M. Rosen, M. Kuno, M. Nirmal, D. J. Norris, and M. G. Bawendi, Phys. Rev. B 54, 4843 (1996).

${ }^{9}$ M. Kuno, J. K. Lee, O. Dabbousi, F. V. Mikulec, and M. G. Bawendi, J. Chem. Phys. 106, 9869 (1997).

${ }^{10}$ O. Labeau, P. Tamarat, and B. Lounis, Phys. Rev. Lett. 90, 257404 (2003).

${ }^{11}$ N. Le Thomas, E. Herz, O. Schöps, and U. Woggon, Phys. Rev. Lett. 94, 016803 (2005).

${ }^{12}$ M. Nirmal, B. O. Dabbousi, M. G. Bawendi, J. J. Mackin, J. K. Trautman, T. D. Harris, and L. E. Brus, Nature (London) 383, 802 (1996).

${ }^{13}$ M. Kuno, D. P. Fromm, H. F. Hamann, A. Gallagher, and D. J. Nesbitt, J. Chem. Phys. 112, 3117 (2000).

${ }^{14}$ M. Kuno, D. P. Fromm, A. Gallagher, D. J. Nesbitt, O. I. Micic, and J. J. Nozik, Nano Lett. 1, 557 (2001).

${ }^{15}$ M. Kuno, D. P. Fromm, S. T. Johnson, A. Gallagher, and D. J. Nesbitt, Phys. Rev. B 67, 125304 (2003).

${ }^{16}$ S. A. Empedocles and M. G. Bawendi, J. Phys. Chem. B 103, 1826 (1999).

${ }^{17}$ K. T. Shimizu, R. G. Neuhauser, C. A. Leatherdale, S. A. Empedocles, 
W. K. Woo, and M. G. Bawendi, Phys. Rev. B 63, 205316 (2001).

${ }^{18}$ K. T. Shimizu, W. K. Woo, B. R. Fisher, H. J. Eisler, and M. G. Bawendi, Phys. Rev. Lett. 89, 117401 (2002).

${ }^{19}$ G. Schlegel, J. Bohnenberger, I. Potapova, and A. Mews, Phys. Rev. Lett. 88, 137401 (2002).

${ }^{20}$ X. Brokmann, J. P. Hermier, G. Messin, P. Desbiolles, J. P. Bouchaud, and M. Dahan, Phys. Rev. Lett. 90, 120601 (2003).

${ }^{21}$ A. Issac, C. von Borczyskowski, and F. Cichos, Phys. Rev. B 71, 161302(R) (2005).

${ }^{22}$ R. Verberk, A. M. van Oijen, and M. Orrit, Phys. Rev. B 66, 233202 (2002).

${ }^{23}$ I. Chung and M. G. Bawendi, Phys. Rev. B 70, 165304 (2004).

${ }^{24}$ J. Wang and P. Wolynes, Phys. Rev. Lett. 74, 4317 (1995).

${ }^{25}$ M. Boguna, A. M. Berezhkovskii, and G. H. Weiss, Physica A 282, 475 (2000).

${ }^{26}$ E. Barkai, Y. Jung, and R. Silbey, Annu. Rev. Phys. Chem. 55, 457 (2004).

${ }^{27}$ G. Margolin and E. Barkai, J. Chem. Phys. 121, 1566 (2004).

${ }^{28}$ O. Flomenbom, J. Klafter, and A. Szabo, Biophys. J. 88, 3780 (2005).
${ }^{29}$ J. Tang and R. A. Marcus, Phys. Rev. Lett. 95, 107401 (2005).

${ }^{30}$ J. Tang and R. A. Marcus, J. Chem. Phys. 123, 054704 (2005).

${ }^{31}$ J. Tang and R. A. Marcus, J. Chem. Phys. 123, 204511 (2005).

${ }^{32}$ P. A. Frantsuzov and R. A. Marcus, Phys. Rev. B 72, 155321 (2005).

${ }^{33}$ H. Lu, L. Xun, and X. S. Xie, Science 282, 1877 (1998).

${ }^{34}$ O. Flomenbom, K. Velonia, D. Loos et al., Proc. Natl. Acad. Sci. U.S.A. 102, 2368 (2005).

${ }^{35}$ M. Hasse, C. G. Hübner, E. Reuther, A. Herrmann, K. Müllen, and T. Basche, J. Phys. Chem. B 108, 10445 (2004).

${ }^{36}$ J. Schuster, F. Cichos, and C. von Borczyskowski, Appl. Phys. Lett. 87, 051915 (2005).

${ }^{37}$ L. Lounis, H. A. Bechtel, D. Gerion, P. Alivisatos, and W. E. Moerner, Chem. Phys. Lett. 329, 399 (2000).

${ }^{38}$ Some errors were found in Table I of Ref. 31. The correct values are $E_{A, 1}=45 \mathrm{meV}, E_{A, 2}=78 \mathrm{meV}, \Gamma_{2} \tau_{2}=1.56,1 / \Gamma_{2}=64 \mathrm{~s}$, and $\lambda=240$ or $5 \mathrm{meV}$.

${ }^{39}$ J. Tang and R. A. Marcus, J. Chin. Chem. Soc. (Taipei) 53, 1 (2006).

${ }^{40}$ H. A. Kramers, Physica (Amsterdam) 7, 284 (1940). 